\title{
L'échotomographie analogique. Etude de la reconstruction acousto-optique à partir de données échographiques
}

\author{
M. DEFONTAINE, M. BERSON*, B. KARLSSON*, F. OSSANT et L. POURCELOT \\ GIP Ultrasons, Bât. Vialle, 2 Bis Bd Tonnellé, 37000 Tours, France \\ *Unité INSERM 316, Bât. Vialle, 2 Bis Bd Tonnellé, 37000 Tours, France
}

\begin{abstract}
résumé : Tomography has been a highly active research field for the last 20 years and great progress has been made in numerical reconstruction methods. This paper deals with a new method of tomographic reconstruction based on optical processing. We first present a system of acquisition by ultrasound and then the backprojection procedure which is performed by optical methods. The projections are coded by an opto-acoustic modulator and deflector into a laser beam and the image is obtained by integrating the resulting pattern over one revolution by a CCD camera. This procedure, which is entirely analogical, allows the real time reconstruction of images, the only delay being the time required for the aquisition ( approximatively $1 \mathrm{sec}$.). Echotomographies obtained by this method are presented.
\end{abstract}

\section{INTRODUCTION}

Actuellement, la tomographie est devenue une technique d'imagerie connue, presque classique. Elle relève de l'imagerie lourde (de $1 \mathrm{MF}$ à $>10 \mathrm{MF}$ par appareil). En effet, le système de reconstruction tomographique dépend, en général, d'une informatique de calcul dédiée, donc onéreuse. De plus, on voit apparaitre la notion de temps de calcul (reconstruction et traitement des images), ce qui prohibe une imagerie temps réel. D'autre part, le dispositif d'acquisition des projections est souvent très encombrant. C'est une technique qui n'a donc pas trouvé d'application dans le milieu des petites et moyennes entreprises. Dans le but de remédier aux inconvénients cités ci-dessus, nous avons approché le problème sous deux nouveaux angles. Tout d'abord le procédé d'acquisition choisi est un dispositif à base d'ultrasons (échotomographie). Les contraintes matérielles qui en découlent (encombrement, coût, danger et utilisation) sont donc minimisées, voire inexistantes. D'autre part, nous avons développé un dispositif de reconstruction analogique. Le principe repose sur la transformation du signal ultrasonore en une information de type optique (reconstruction instantanée). La seule limitation dans le temps est celle de la durée d'acquisition.

Dans un premier temps, nous rappelons le principe physique de la tomographie et nous replaçons l'échotomographie dans le contexte de l'imagerie. Dans un deuxième temps, nous nous attachons à donner une description d'abord globale de notre prototype d'échotomographie, et ensuite nous nous intéressons plus précisèment à la chaîne de reconstruction acousto-optique (RAO). La dernière partie est consacrée à la validation expérimentale de ce système d'échotomographie. 


\section{L'ECHOTOMOGRAPHIE.}

\subsection{Principe physique de la tomographie}

C'est une technique d'imagerie qui permet d'obtenir des coupes dans un objet [1]. Elle se divise en deux parties distinctes. La première concerne le dispositif d'acquisition. Il se compose en général d'un ensemble source/détecteur qui se déplace autour du corps à tomographier. A chaque pas de rotation, l'objet est noyé dans le champ d'émission de la source. Le schéma de la fig. 1 décrit la configuration d'acquisition en échotomographie.

Pour chaque angle de visée, les données acquises forment une projection. La seconde partie relève de la reconstruction par rétroprojection (figure 2) [2].

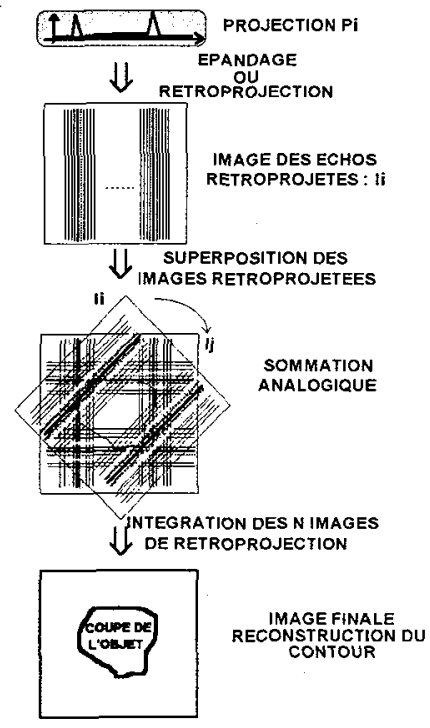

Fig. 2. Illustration du principe physique de la reconstruction par rétroprojection.

\subsection{Principe de l'échotomographie}

Comme nous pouvons l'observer sur le schéma de la figure 3 , chaque donnée de la projection obtenue en échotomographie est le résultat d'une intégration des échos dans une direction perpendiculaire à la direction d'émission. L'acquisition en échotomographie est à " $90^{\circ}$ " par rapport à celle en tomo X.

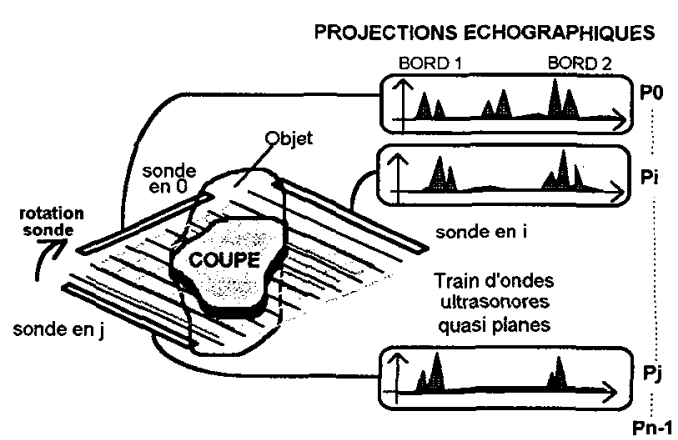

Fig. 1. Dispositif d'acquisition des projections en échotomographie.

Chaque donnée de la projection Pi est épandue ou rétroprojetée sur toute une ligne. Ceci présuppose une propagation des ultrasons en ligne droite. Ce n'est pas le cas lorsque l'onde traverse des structures qui présentent de brusques variations de géométrie et d'impédance acoustique. C'est la limitation principale de la méthode.

On obtient une image de rétroprojection Ii. On recherche la valeur de la fonction $f(x, y)$ (réflectivité) en tout point $(x, y)$ de l'image.

Lorsque toutes les images de rétroprojection ont été formées et positionnées conformément à leur incidence d'acquisition, on réalise la sommation en tout point $(\mathrm{x}, \mathrm{y})$ des $\operatorname{Ii}(\mathrm{x}, \mathrm{y})(0<\mathrm{i}<\mathrm{n}, \mathrm{n}=$ nombre de projections).

Le résultat représente $f(x, y)$ (sans filtrage).

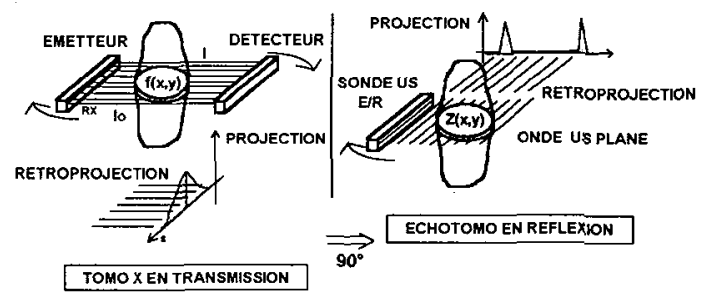

Fig. 3. Comparaison des principes de tomoX en transmission et de l'échotomographie. 


\section{DESCRIPTION DU DISPOSITIF D'ECHOTOMOGRAPHIE ANALOGIQUE.}

La figure 4 illustre les différents blocs fonctionnels du prototype.

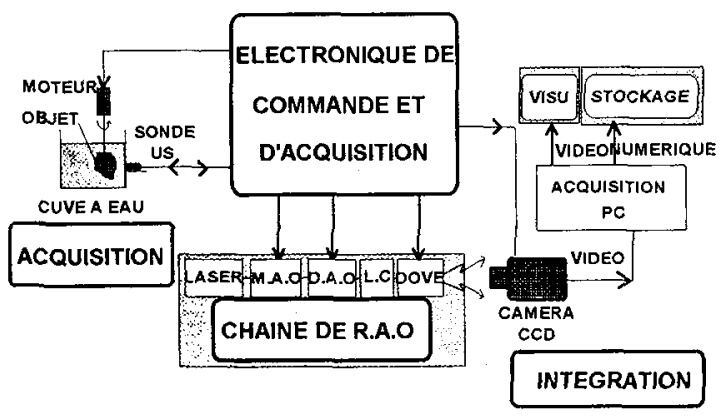

Fig.4. Schéma synoptique
- Ce schéma comporte une partie "électronique de commande". Elle effectue la gestion de l'émission/réception au niveau du transducteur, génère les signaux nécessaires à la chaîne de reconstruction acousto-optique (RAO), et synchronise l'ensemble du dispositif.

- La partie "acquisition" comprend une sonde à 3.5 $\mathrm{MHz}$ qui génère une onde plane focalisée mécaniquement à $7 \mathrm{~cm}$.

- La "chaîne de reconstruction" est développée au chapitre suivant.

- La fonction "intégration" des images de rétroprojection est assurée par une caméra CCD.

- Le bloc "Acquisition" permet le stockage et la visualisation de l'image reconstruite sur PC.

\section{LA CHAINE DE RECONSTRUCTION ACOUSTO-OPTIQUE}

Lors de la reconstruction analogique par rétroprojection, le support de l'information échographique est un LASER HeNe émettant dans le rouge $(633 \mathrm{~nm}, 5 \mathrm{~mW}$ ). (figure 5 )

- Un modulateur acousto-optique (MAO) module l'intensité du faisceau LASER en fonction de l'amplitude du signal échographique (projection);

- Une optique de grossissement adapte le faisceau au fonctionnement du déflecteur.;

- Le déflecteur acousto-optique (DAO) balaye le spot pendant une durée correspondant à la réception des échos (signal d'une projection);

- La lentille cylindrique assure l'épandage du spot;

- La rotation des images de rétroprojection est réalisée par le prisme de Dove;

- La caméra intègre durant une révolution de l'objet.

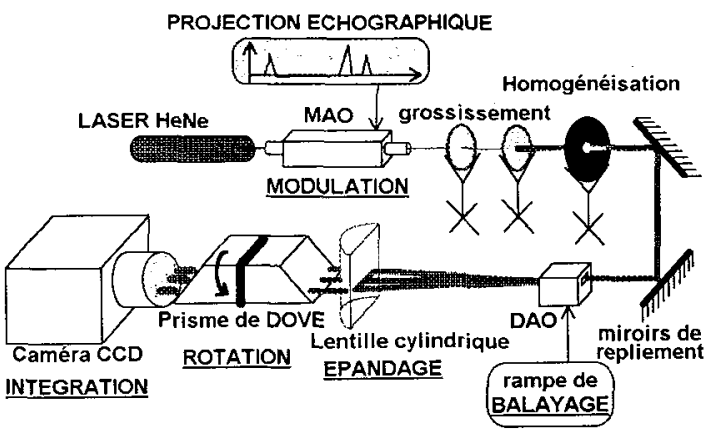

Fig. 5. Dispositif de reconstruction acousto-optique

\section{PRESENTATION DES PREMIERES ECHOTOMOGRAPHIES ANALOGIQUES.}

\subsection{Présentations des résultats}

La validation de cet échotomographe analogique a été effectuée en plusieurs étapes. Nous avons commencé par tester la reconstruction à l'aide de fantômes en polystyrène. Les ultrasons sont presque totalement réfléchis sur la première interface eau/objet de ce matériau. Ce sont donc des images de contours que nous avons obtenues. Les premiers fantômes ont été élaborés avec des formes continues (cylindre, ellipse), puis on a compliqué de plus en plus leur géométrie (carré, losange, triangle), jusqu'à des contours presque discontinus (forme en étoile). C'est l'image de l'étoile que nous présentons sur la figure 7. La seconde étape a consisté à élaborer des fantômes à base d'agar-agar dopés à l'oxyde d'aluminium. Ces objets présentent des inclusions dont les formes géométriques sont connues. Les deux textures diffèrent au niveau du dopage en alumine (figure 8 ). 


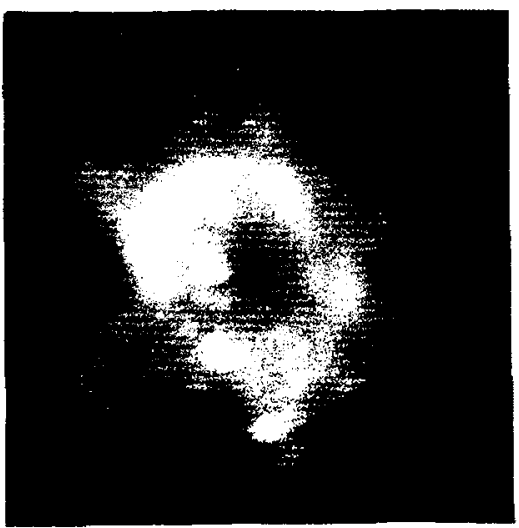

Fig. 7. Reconstruction d'une étoile en polystyrène.

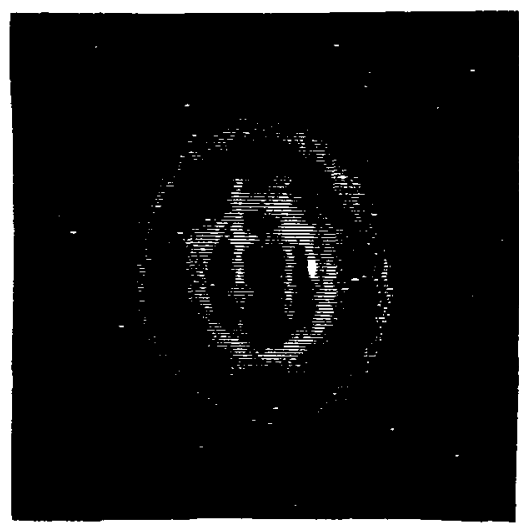

Fig. 8. Reconstruction d'un fantôme d'agar-agar avec inclusion.

\subsection{Discussions}

Il faut noter qu'aucun traitement n'a été effectué sur ces reconstructions. En numérique, la reconstruction par rétroprojection nécessite un filtrage (filtre rampe appliqué aux T,F des projections). Ce sera une autre étape de ce projet. Par ailleurs, l'échotomographie d'objets aux formes anguleuses et présentant plusieurs facettes à l'onde incidente, pose des problème de réflexions omnidirectionelles et de diffraction sur les pointes. Ces phénomènes tendent à bruiter la reconstruction. C'est le cas de l'objet tomographié sur la figure 7 , l'étoile. On reconnait cependant les formes extérieures de celle-ci. Il est nécessaire de quantifier ces problèmes, c'est le but d'une prochaine étape [3]. L'échotomographie de la figure 8. a été réalisée en champ lointain. Dans cette situation les centres de rotation objet et image ne coîncident plus. Aussi, les rapports de distance sur la coupe ne sont plus vérifiés après reconstruction. C'est un problème de sensibilité qui nous a amené à positionner les objets hörs zone focale. Cependant, dans cette situation un problème d'homogénéité de l'épaisseur de coupe apparait. Une amélioration consisterait à fabriquer une sonde composée de plusieurs éléments dans la direction de l'épaisseur, et d'effectuer alors une focalisation électronique. D'autre part, il serait intéressant d'ajouter un système d'amplification logarithmique, ou de correction de gain selon la profondeur, sur la réception.

\section{CONCLUSION}

Ce protype d'échotomographie analogique donne des résultats fidèles à la réalité des objets testés. La validation expérimentale n'est pas achevée. Entre autres, les fantômes testant la résolution n'ont pas été élaborés. Une version dégradée de cet appareil (tomo de contours) peut être envisagée. Dans ce contexte, on peut effectuer des estimations de volumes dans des temps très courts.

\section{REFERENCES}

[1] Dines K. A., Goss S, A., Computed ultrasonic reflection tomography, IEEE on ultrasonics, ferroelectrics and frequency control, vol. 3, may 1987, pp. 309-318.

[2] Itti R., Pourcelot L., Simeon G., Dispositif de reconstriction tomographique instantanée par rétroprojection, J. Fr. Biophysique et Med. Nucl., 3, 5, 1979, pp. 249-252.

[3] Harris R. A., Follet D. H., Halliwell M., Wells P.N.T., Ultimate limits in ultrasonic imaging resolution, Ultrasound in Medecine and Biology, vol 17, N6, 1991, pp. 309-318. 